\title{
Excited hadrons in $n_{f}=2$ QCD
}

\section{G. Engel, ${ }^{a}$ C. Gattringer, ${ }^{a}$ C. B. Lang ${ }^{* a}{ }^{a}$ M. Limmer, ${ }^{a}$ D. Mohler,${ }^{a}$ and A. Schäfer ${ }^{b}$}

${ }^{a}$ Inst. f. Physik, FB Theoretische Physik, Karl-Franzens-Universität Graz, Graz, Austria

${ }^{b}$ Inst. f. Theoret. Physik, Univ. Regensburg, Regensburg, Gernmay

E-mail: georg.engel@uni-graz.at, christof.gattringer@uni-graz.at, christian.lang@uni-graz.at, markus.limmereuni-graz.at,

daniel.mohler@uni-graz.at, andreas.schaefer@physik.uni-regensburg.de

The chirally improved (CI) fermion action allows us to obtain results for pion masses down to $320 \mathrm{MeV}$ on (in lattice units) comparatively small lattices with physical extent of $2.4 \mathrm{fm}$. We use differently smeared quarks sources to build sets of several interpolators. The variational method then leads to excellent ground state masses for most mesons and baryons. The excited state signals weaken in quality towards smaller quark masses. In particular the excited baryons come out too high.

The XXVII International Symposium on Lattice Field Theory - LAT2009

July 26-31 2009

Peking University, Beijing, China

${ }^{*}$ Speaker. 


\section{Study with two dynamical CI fermions}

We are presenting results of a hadron mass spectrum calculation with emphasis on possible identification of excited states. The gauge configurations have been obtained with dynamical, mass degenerate up and down quarks. For the fermions we used the chirally improved (CI) Dirac operator $D_{C I}[1,2]$. This is a parameterized fermion action of the form

$$
D_{m n}=\sum_{\alpha=1}^{16} \Gamma_{\alpha} \sum_{p \in \mathscr{P}{ }_{m, n}^{\alpha}} c_{p}^{\alpha} \prod_{l \in p} U_{l} \delta_{n, m+p}
$$

where $\mathscr{P}_{m, n}^{\alpha}$ symbolizes paths from site $m$ to $n$. Inserting the ansatz in the Ginsparg-Wilson (GW) equation, truncating the length of the contributions (to, e.g., distance 4), and comparing the coefficients, leads to a set of algebraic equations, which can be solved (norm minimization). We used a truncated action with terms involving coupling to neighboring sites within a $3^{4}$ hypercube plus some extra terms, giving rise to several hundred terms. Part of our definition of the Dirac action is one step of stout smearing [3] of the gauge configuration. The eigenvalues of $D_{C I}$ are closer to the unit circle (where the eigenvalues of exact GW-operators are located) than those of, e.g., the improved Wilson operator for the same lattice size (in lattice units). However, (lattice) chiral symmetry is still violated, albeit to a smaller amount. The gauge action used is a tadpole-improved Lüscher-Weisz action.

The dynamics was implemented with the hybrid Monte Carlo (HMC) algorithm with Hasenbusch mass preconditioning (with two pseudofermions) and a chronological inverter utilizing the mixed precision technique [4]. Details and parameters of the action and the methods of simulation can be found in [5]. There one also finds a discussion of equilibration and determination of lattice spacing and AWI (PCAC) mass of the quarks.

The analysis presented here is based on three ensembles of gauge configurations for lattice size $16^{3} \times 32$, with parameters summarized in Table 1 .

\begin{tabular}{crrrrrrr}
\hline \hline set & $\beta_{L W}$ & $a m_{0}$ & $t_{M D}$ & configs. & $a[\mathrm{fm}]$ & $m_{\pi}[\mathrm{MeV}]$ & $m_{A W I}[\mathrm{MeV}]$ \\
\hline A & 4.70 & -0.050 & 600 & 100 & $0.151(2)$ & $525(7)$ & $42.8(4)$ \\
B & 4.65 & -0.060 & 1200 & 200 & $0.150(1)$ & $470(4)$ & $34.1(2)$ \\
C & 4.58 & -0.077 & 1200 & 200 & $0.144(1)$ & $322(5)$ & $15.3(4)$ \\
\hline
\end{tabular}

Table 1: Overview of the three ensembles of gauge configurations on which this analysis is based. The parameters given are: gauge coupling, bare mass parameter $a m_{0}$, number of molecular dynamics (MD) time units, number of analyzed configurations (in equilibrium and each separated by 5 MD units), lattice spacing $a$ determined from the static potential with Sommer parameter $0.48 \mathrm{fm}$, pion mass, AWI mass (from the PCAC relation). For further details see [5].

\section{Variational analysis and hadron interpolators}

In the variational method [6] one studies the cross-correlation matrix of several lattice operators $O_{i}$ with the correct quantum numbers. Inserting a complete set of states (and assuming a 
discrete spectrum, which is always the case for finite lattices) one finds a superposition of exponentially decaying contributions,

$$
C(t)_{i j}=\left\langle O_{i}(t) \overline{O_{j}}(0)\right\rangle=\sum_{n}\left\langle 0\left|O_{i}\right| n\right\rangle\left\langle n\left|O_{j}^{\dagger}\right| 0\right\rangle \mathrm{e}^{-t M_{n}} .
$$

Assuming that we have a complete enough set of operators the solution of the generalized eigenvalue problem

$$
C(t) \vec{v}_{i}=\lambda_{i}(t) C\left(t_{0}\right) \vec{v}_{i}
$$

allows one to disentangle the individual states and the corresponding energies,

$$
\lambda_{i}(t) \propto \mathrm{e}^{-t M_{i}}\left(1+\mathscr{O}\left(\mathrm{e}^{-t \Delta M_{i}}\right)\right) .
$$

The eigenvectors are "fingerprints" of the states which one may follow through several time slices in order to ensure that the state has been identified consistently (see also the discussion in [7].)

In order to increase the number of hadron operators and to improve the correlation signal quality we built interpolating fields with different smearing of the quark fields. We used Jacobi smeared quark sources, e.g., $u_{s} \equiv S_{s} u$, with an hermitian smearing operator $S_{s}$ as discussed in [5], with parameters adjusted to produce two different smearing widths, a wide source ( $s=w$, radius $0.55 \mathrm{fm})$ and a narrow source $(s=n$, radius $0.27 \mathrm{fm})$.

We also used derivative quark sources as discussed in [8], e.g., sources like $u_{\partial_{k}}=D_{k} S_{w} u$, where $k$ denotes the spatial direction of the covariant derivative

$$
D_{i}(\vec{x}, \vec{y})=U_{i}(\vec{x}, 0) \delta(\vec{x}+\hat{i}, \vec{y})-U_{i}(\vec{x}-\hat{i}, 0)^{\dagger} \delta(\vec{x}-\hat{i}, \vec{y}) .
$$

The interpolating field operators are built on (in the 3D time slices) 3 times HYP smeared gauge configurations [9] with smeared valence quark sources $u_{s}, d_{s}$ (and the strange quark $s_{s}$ ). We regularly shift the center of the sources when passing from one configuration to the next in order to improve decorrelation.

For the meson operators we use bilinears. Depending on the quantum numbers this allows for sets of operators with different Dirac structure and varying spatial extent. As an example, for the pseudoscalar meson we have

$$
\begin{array}{r}
\bar{u}_{n} \gamma_{5} d_{n}, \bar{u}_{n} \gamma_{5} d_{w}, \bar{u}_{w} \gamma_{5} d_{w}, \bar{u}_{n} \gamma_{t} \gamma_{5} d_{n}, \bar{u}_{n} \gamma_{t} \gamma_{5} d_{w}, \bar{u}_{w} \gamma_{t} \gamma_{5} d_{w}, \\
\bar{u}_{\partial_{i}} \gamma_{i} \gamma_{5} d_{n}, \bar{u}_{\partial_{i}} \gamma_{i} \gamma_{5} d_{w}, \bar{u}_{\partial_{i}} \gamma_{i} \gamma_{t} \gamma_{5} d_{n}, \bar{u}_{\partial_{i}} \gamma_{i} \gamma_{t} \gamma_{5} d_{w}, \bar{u}_{\partial_{i}} \gamma_{5} d_{\partial_{i}}, \bar{u}_{\partial_{i}} \gamma_{t} \gamma_{5} d_{\partial_{i}} .
\end{array}
$$

The nucleon interpolators have the form

$$
N^{(i)}=\varepsilon_{a b c} \Gamma_{1}^{(i)} u_{a}\left(u_{b}^{T} \Gamma_{2}^{(i)} d_{c}-d_{b}^{T} \Gamma_{2}^{(i)} u_{c}\right)
$$

with the choices $\left(\Gamma_{1}^{(i)}, \Gamma_{2}^{(i)}\right)=\left(\mathbf{1}, C \gamma_{5}\right),\left(\gamma_{5}, C\right)$, and (i1, $\left.C \gamma_{4} \gamma_{5}\right)$ for $i=1,2,3$ respectively, where $u$, $d$ denote again smeared quarks. The $\Delta$ interpolator is

$$
O_{\Delta, k}=\varepsilon_{a b c} u_{a}\left(u_{b}^{T} C \gamma_{k} u_{c}\right), \quad k=1,2,3 .
$$

projected to spin $\frac{3}{2}$. The baryon propagators are projected to definite parity. 

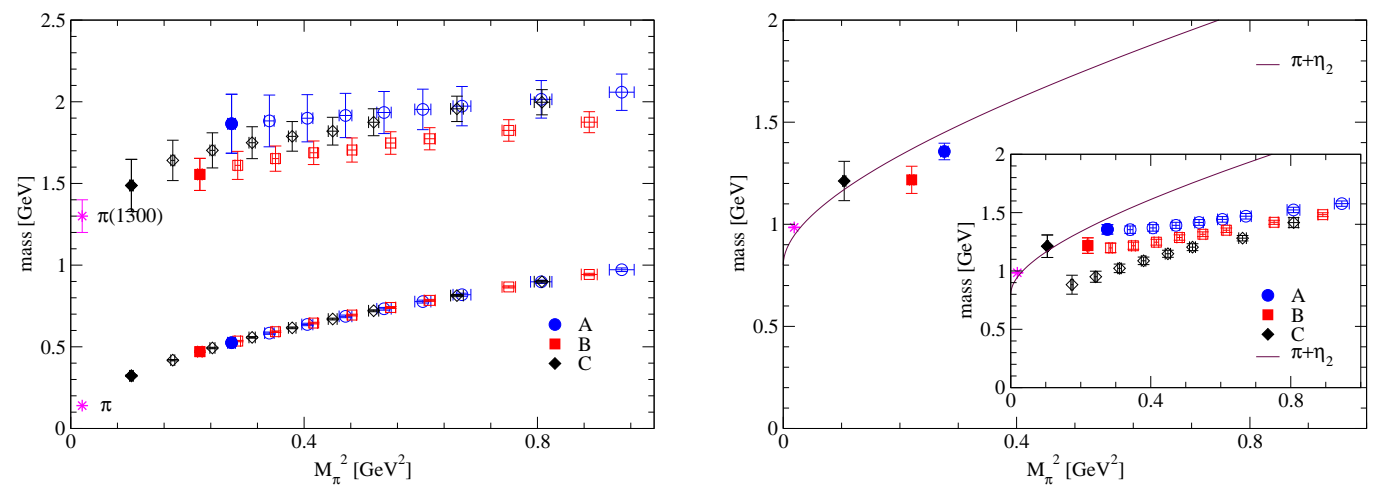

Figure 1: L.h.s.: The ground state pion and its first excitation. Open symbols denote partially quenched data, the full symbols denote the dynamical data for the three ensembles A, B and C. R.h.s.: The dynamical data for the $0^{++}$channel $a_{0}$ are compared with the energy level expected for a $\pi-\eta_{2}$ channel. In the insert also the partially quenched data (open symbols) are shown, as discussed in the main text.

\section{Results for mesons}

$\mathbf{0}^{-+}: \pi^{ \pm}(\mathbf{1 4 0}), \pi^{ \pm}(\mathbf{1 3 0 0})$. As discussed in [5], in the multi-operator (variational) analysis at small pion masses the backwards running (in time) pion limits the observation range for the excited state. This can be cured by a larger time-size; however, for physical pion masses we expect that one needs at least $N_{t}=64$ for lattice spacing $a=0.15 \mathrm{fm}$ or $N_{t}=128$ for $a=0.075 \mathrm{fm}$.

We choose the fit interval of the exponential fit to the non-leading eigenvalues based on the window where the backwards running contribution is not yet dominant and get the excited pion signal in Fig. 1 (left). We include in this plot (as in some of the other figures) the results for partially quenched data, i.e., where the valence quark masses are larger than the sea quark masses.

$\mathbf{0}^{++}: \mathbf{a}_{\mathbf{0}}(\mathbf{9 8 0}), \mathbf{a}_{\mathbf{0}}(\mathbf{1 4 5 0})$. The isovector, scalar meson has led to controversial results in lattice simulations $[10,11]$. Most quenched studies found a ground state extrapolating towards the mass of the $a_{0}(1450)$ for smaller valence quark masses. Results for (two) dynamical quarks seem to lead to smaller masses, compatible with an extrapolation towards the $a_{0}(980)$. However, for these masses the energy values are close to those of an expected $\pi-\eta_{2}$ channel in s-wave (mass of $\eta_{2}$ estimated [12]). Fig. 1 (r.h.s.) exhibits the situation.

When also plotting partially quenched values, we find an interesting effect for the ensemble C with the smallest sea quark mass. The partially quenched data do not smoothly extrapolate to the dynamical point. An explanation has been offered in [13]: the partially quenched states may couple to pairs of pseudoscalars (composed of valence and sea quarks), leading to unphysical contributions that cancel in the fully dynamical case.

Also, we find a broad range of values for the extracted energy levels for different sets of contributing interpolators. The issue, whether the $a_{0}$ ground state is dominantly a tetraquark state, is still not settled (see [14]).

$\mathbf{1}^{--}: \rho^{ \pm}(\mathbf{7 7 0}), \rho^{ \pm}(\mathbf{1 4 5 0})$. In Fig. 2 (1.h.s.) we show the results for ground state and first excitation in the $\rho$-channel. For a better signal we allow for different operator combinations for the excited states. The broken lines give an error window for linear extrapolating fits based on the three 

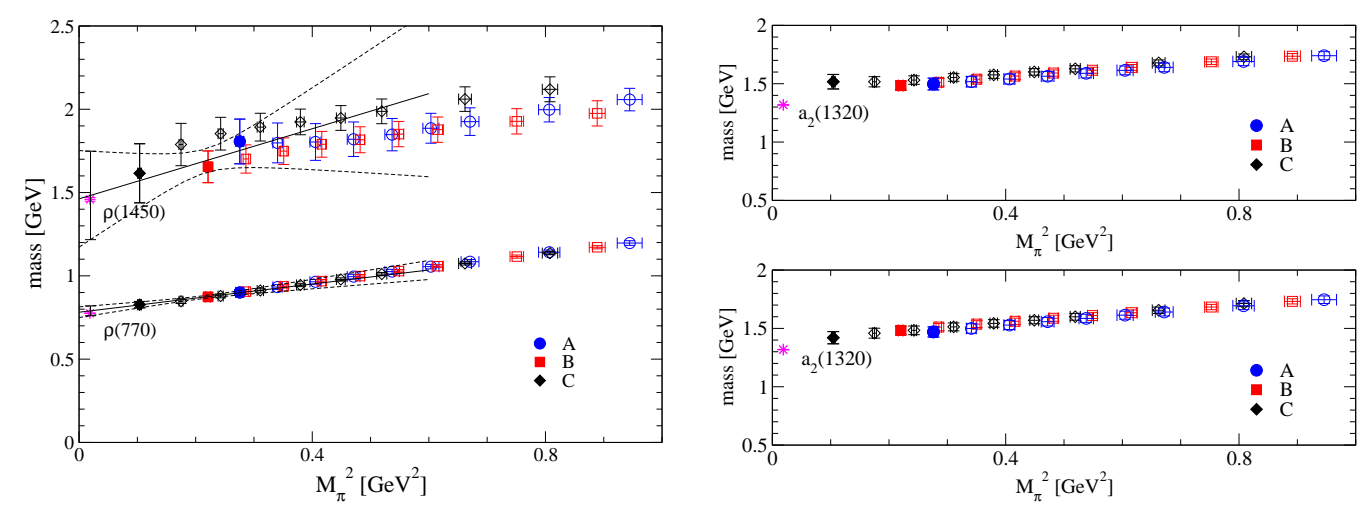

Figure 2: L.h.s.: Ground and excited state in the $1^{--}$channel. Open symbols denote partially quenched results. R.h.s.: Ground state in the $2^{++}$channel separately evaluated for interpolator sets in the $E$ (top) and the $T_{2}$ representation (below) of the cubic group. Filled symbols denote the values from the three fully dynamical data sets, open symbols show partially quenched results.

dynamical points only. The agreement with the experimental values is surprisingly good in view of the $\rho$ being a resonance; however, the decay pions are in relative $\mathrm{p}$-wave and due to the given lattice size the necessary extra unit of momentum stabilizes the vector meson.

We find more admixtures of still higher excitations, and for some combinations of operators we can identify a 3 rd energy level compatible with the $\rho(1700)$.

Our data in the exotic $1^{-+}$channel is too noisy to allow extraction of a ground state energy level.

$\mathbf{1}^{++}: \mathbf{a}_{\mathbf{1}}(\mathbf{1 2 6 0})$ and $\mathbf{1}^{+-}: \mathbf{b}_{\mathbf{1}}(\mathbf{1 2 3 5})$. In both channels we see reliable signals only when including derivative interpolators (cf. the discussion for the quenched case in [8]). The error bars are somewhat larger for $1^{+-}$than for $1^{++}$, where they are roughly $\pm 50 \mathrm{MeV}$. In both cases a linear extrapolation points towards the experimental value (within the errors). The mass difference $m_{\text {latt }, b_{1}}-m_{\text {latt }, a_{0}}$ is approximately $200 \mathrm{MeV}$.

$\mathbf{2}^{++}: \mathbf{\mathbf { a } _ { 2 }}(\mathbf{1 3 2 0})$. In this channel two representations of the cubic group couple: $E$ and $T_{2}$. Again interpolators involving derivative sources are necessary, e.g., $\varepsilon_{i j k} \bar{u}_{\partial_{i}} \gamma_{j} d_{n}$. We find good signals only when we include several interpolators and thus this is a case where the variational method is crucial. Fig. 2 (r.h.s.) demonstrates the situation. The results for the independent analysis of both representations are compatible, although $T_{2}$ appears to extrapolate better to the physical value.

$\mathbf{2}^{-+}: \pi_{\mathbf{2}}(\mathbf{1 6 7 0})$ and $\mathbf{2}^{--}: \rho_{\mathbf{2}}(\mathbf{1 9 4 0})$ ?. Qualitatively the situation is like for the $2^{++}$channel. In both representations we find values compatible with each other and within large errors of order 200 $\mathrm{MeV}$ also (in linear extrapolation) with the physical mass value.

\section{Results for baryons}

$\frac{1}{2}^{+}: \mathbf{N}(\mathbf{9 4 0}), \mathbf{N}(\mathbf{1 4 4 0}), \mathbf{N}(\mathbf{1 7 1 0})$. The baryon system poses various challenges in the excited sector, among them the identification of the Roper state in lattice calculations. In most calculations in 

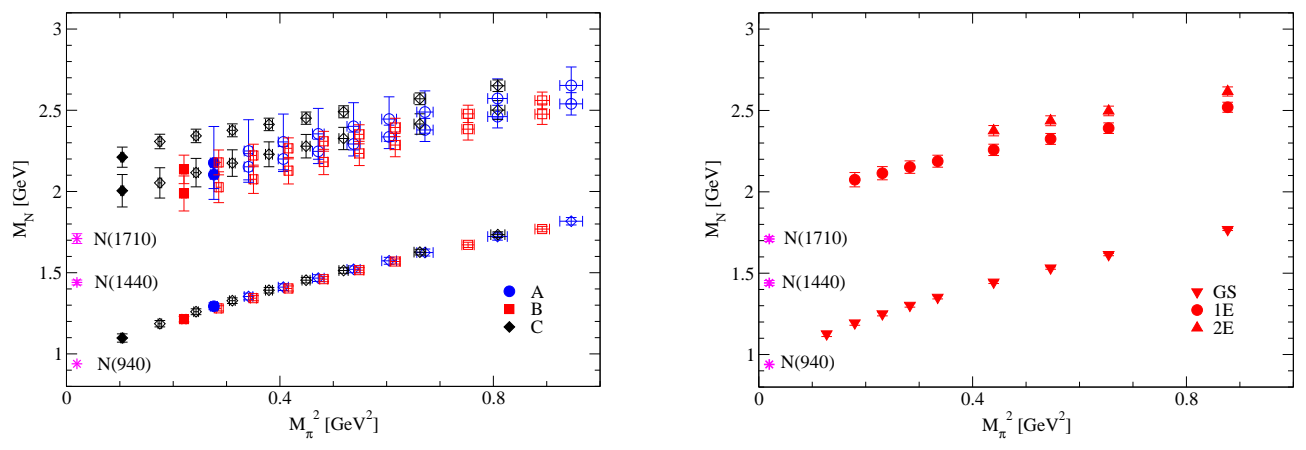

Figure 3: Results for the positive parity nucleon channel for the new data with dynamical fermions (1.h.s.) compared to earlier results from quenched configurations [15] (r.h.s.).

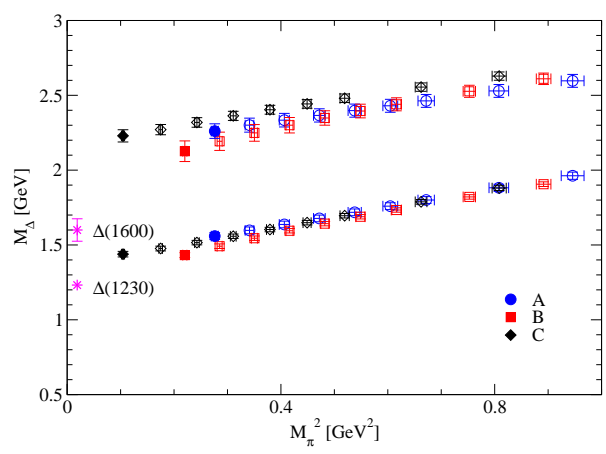

Figure 4: Positive parity: Ground state $\Delta$ and first excitation.

the quenched approximation the first excitation of the (positive parity) nucleon came out too high, and only within large error bars an extrapolation towards the experimental Roper mass was imaginable (see, e.g., the quenched study in [15]). Only recently, and at this conference [16, 17], there are results showing an energy level extrapolating towards smaller masses. That study was based on optimizing combinations of interpolators with several widths, like in this and earlier work [18].

There was some hope that introducing the quark dynamics might improve the situation. Our present analysis does not strengthen that hope (Fig. 3). Indeed our new results for dynamical fermions are similar to earlier quenched results [15]. We want to emphasize that it is important in this case to simultaneously find both excitations (the Roper and the state extrapolating to the $N(1710)$ ) in order to have a convincing identification. Both excited states are too high in our results which might indicate that the given volume is too small for baryon excitations.

$\frac{1}{2}^{-}: \mathbf{N}(\mathbf{1 5 3 5}), \mathbf{N}(\mathbf{1 6 5 0})$. We clearly identify two states but cannot quantify their mass splitting due to large errors.

$\frac{3}{2}^{+}: \Delta(\mathbf{1 2 3 2}), \Delta(\mathbf{1 6 0 0})$ and $\frac{3}{2}^{-}: \Delta(\mathbf{1 7 0 0})$. In the positive parity sector (see Fig. 4) we find that the ground state is closer to the experimental values, as compared to the quenched results. The excited state is clearly seen, but again too high, maybe due to volume squeezing. The ground state in the negative parity sector is clearly identified and extrapolates towards the experimental mass. 
Thus, while our meson results look quite good, the excited baryons come out too high. Improvement of the signal might be obtained with larger lattices and possibly extending the set of interpolator (see, e.g., [19]). Further results for the axial charge of baryons including baryons with strangeness are discussed in [20].

Acknowledgment: This work has been supported by Austrian FWF DK W1203-N08 and German DFG project SFB/TR-55. The calculations have been performed on the SGI Altix 4700 of the Leibniz-Rechenzentrum Munich and on local clusters at ZID at the University of Graz.

\section{References}

[1] C. Gattringer, Phys. Rev. D 63 (2001) 114501 [hep-lat/ 0003005 ].

[2] C. Gattringer, I. Hip and C. B. Lang, Nucl. Phys. B 597 (2001) 451 [hep-lat/ 0007042 ].

[3] C. Morningstar and M. Peardon, Phys. Rev. D 69 (2004) 054501 [hep-lat/ 0311018 ].

[4] S. Dürr, Z. Fodor, C. Hoelbling, R. Hoffmann, S. Katz, S. Krieg, T. Kurth, L. Lellouch, T. Lippert, K. Szabo and G. Vulvert, Phys. Rev. D 79 (2008) 014501 [arXiv: 0802.2706 [hep-lat]].

[5] C. Gattringer, C. Hagen, C. B. Lang, M. Limmer, D. Mohler and A. Schäfer, Phys. Rev. D 79 (2009) 054501 [0812.1681].

[6] C. Michael, Nucl. Phys. B 259 (1985) 58. M. Lüscher and U. Wolff, Nucl. Phys. B 339 (1990) 222.

[7] B. Blossier, M. DellaMorte, G. von Hippel, T. Mendes and R. Sommer, JHEP 0904 (2009) 094 [0902.1265].

[8] C. Gattringer, L. Y. Glozman, C. B. Lang, D. Mohler and S. Prelovsek, Phys. Rev. D 78 (2008) 034501 [arXiv:0802.2020 [hep-lat]].

[9] A. Hasenfratz and F. Knechtli, Phys. Rev. D 64 (2001) 034504 [arXiv: hep-lat/ 010302 9].

[10] C. McNeile, $P o S$ (LATTICE2007) (2007) 019 [arXiv:0710.0985 [hep-lat]].

[11] K. Jansen, C. McNeile, C. Michael and C. Urbach, (2009) [arXiv:0906.4720 [hep-lat]].

[12] K. Jansen, C. Michael and C. Urbach, Eur. Phys. J. C 58 (2008) 261 [arXiv: 0804.3871 [hep-lat]].

[13] S. Prelovsek, C. Dawson, T. Izubuchi, K. Orginos and A. Soni, Phys. Rev. D 70 (2004) 094503 [hep-lat/0407037].

[14] S. Prelovsek et. al., contribution to this conference, PoS (LAT2009) (2009) 088.

[15] T. Burch, C. Gattringer, L. Y. Glozman, C. Hagen, D. Hierl, C. B. Lang and A. Schäfer, Phys. Rev. D 74 (2006) 014504 [hep-lat/ 0604019 ].

[16] M. S. Mahbub, A. O. Cais, W. Kamleh, B. G. Lasscock, D. B. Leinweber and A. G. Williams, Phys. Rev. D 80 (2009) 054507 [arXiv: 0905.3616 v1 [hep-lat] ].

[17] S. Mahbub et. al., contribution to this conference, PoS (LAT2009) (2009).

[18] T. Burch, C. Gattringer, L. Y. Glozman, R. Kleindl, C. B. Lang and A. Schäfer, Phys. Rev. D 70 (2004) 054502 [hep-lat/ 0405006$].$

[19] M. Peardon, J. Bulava, J. Foley, C. Morningstar, J. Dudek, R. G. Edwards, B. Joo, H.-W. Lin, D. G. Richards and K. J. Juge, (2009) [arXiv:0905.2160 [hep-lat] ].

[20] D. Mohler et. al., contribution to this conference, PoS (LAT2009) (2009). 